PHYSICAL REVIEW E 85, 046111 (2012)

\title{
Approximate entropy of network parameters
}

\author{
James West* \\ Statistical Cancer Genomics, Paul O'Gorman Building, UCL Cancer Institute and Department of Physics \& Astronomy, \\ University College London, London, UK \\ Lucas Lacasa ${ }^{\dagger}$ \\ Departamento de Matemática Aplicada y Estadística, ETSI Aeronáuticos, Universidad Politécnica de Madrid, Madrid, Spain and \\ Department of Physics, Clarendon Laboratory, University of Oxford, Oxford, UK \\ Simone Severini ${ }^{\ddagger}$ \\ Department of Computer Science and Department of Physics \& Astronomy, University College London, London, UK \\ Andrew Teschendorff ${ }^{\S}$ \\ Statistical Cancer Genomics, Paul O'Gorman Building, UCL Cancer Institute, London, UK \\ (Received 11 January 2012; published 19 April 2012)
}

\begin{abstract}
We study the notion of approximate entropy within the framework of network theory. Approximate entropy is an uncertainty measure originally proposed in the context of dynamical systems and time series. We first define a purely structural entropy obtained by computing the approximate entropy of the so-called slide sequence. This is a surrogate of the degree sequence and it is suggested by the frequency partition of a graph. We examine this quantity for standard scale-free and Erdös-Rényi networks. By using classical results of Pincus, we show that our entropy measure often converges with network size to a certain binary Shannon entropy. As a second step, with specific attention to networks generated by dynamical processes, we investigate approximate entropy of horizontal visibility graphs. Visibility graphs allow us to naturally associate with a network the notion of temporal correlations, therefore providing the measure a dynamical garment. We show that approximate entropy distinguishes visibility graphs generated by processes with different complexity. The result probes to a greater extent these networks for the study of dynamical systems. Applications to certain biological data arising in cancer genomics are finally considered in the light of both approaches.
\end{abstract}

DOI: 10.1103/PhysRevE.85.046111

PACS number(s): $89.75 . \mathrm{Hc}$, 89.75.Fb, 89.70.Cf

\section{INTRODUCTION}

Concepts such as information, entropy, and measures of complexity are highly connected topics within the core of dynamical systems and chaos theory. The area benefits from a wealth of literature that dates back to the works of Kolmogorov on metric entropies, and continues with the developments of Sinai, Eckmann, Ruelle, and others (see [1] and references therein for a review on the topic). Roughly speaking, this branch of science is relatively mature for answering questions such as how a system which is sensitive to initial conditions (with positive characteristic Lyapunov exponents) generates uncertainty as time evolves, and how this entropy production is related to the structure (invariant measure) of the system.

In recent years, in parallel with the advent of the study of complex networks (see, for example, [2,3]), similar ideas aiming to describe the amount of organization of these systems have started to take root. As a matter of fact, to describe mathematically the amount of heterogeneity and complexity found in natural and technological networks is nowadays a major endeavor in the frameworks provided by network theory, general data analysis, and inference. Several recent

\footnotetext{
*jawest@gmail.com

†lucas.lacasa@upm.es

${ }^{\ddagger}$ simoseve@gmail.com

§a.teschendorff@ucl.ac.uk
}

works point toward an entropic origin for a variety of key properties of complex networks that we find around us, such as the biodiversity maintenance in ecological networks [4,5], or, more generally, the emergence of robust degree-degree correlations [6] and communities in social and biological networks [7]. Indeed, the amount of heterogeneity in a network is a basic ingredient for quantifying properties of diffusion processes, such as the spread of human epidemics and computer viruses [8-10].

Some theoretical approaches to deal with the notion of network heterogeneity include Refs. [11,12], where a statistical mechanics perspective is adopted to estimate the (thermodynamic) entropy of network ensembles given by a set of constraints. Other lines of research make use of spectral theory to derive optimal network configurations [13-15]. However, the majority of the proposed network-based entropic functionals are, so far, entropies à la Shannon (see also [16] for a recent review). Less work has been reported on the extension of other invariant measures to the network theoretic context (see however [17]).

To begin filling the gap, we consider the notion of approximate entropy (often denoted ApEn) as introduced by Pincus [18]. ApEn is a finite-size statistic of the Eckmann-Ruelle entropy originally proposed as a measure of the complexity of a system changing in time. At present, time series are the main area of application. We explore network-based extensions of approximate entropy and ask questions about the usefulness 
of this parameter in estimating the degrees of uncertainty both in the case of static and growing networks. It is important to see that any attempt of defining an approximate entropy of network parameters (following along the same lines as Pincus) must be based on some ordering of the data. As a starting point, it is natural to consider certain orderings associated with the degree sequence and its related quantities.

The present work contains several contributions: (1) The general point is the novelty of studying the notion of approximate entropy in a network theoretic context; (2) this requires the translation of network parameters into a time series that is amenable to be investigated with analytic tools; accordingly, we define a binary string associated with the degree sequence as is suggested by the notion of frequency partition; (3) as expected, given that the string reflects some coarse-grained properties of the degree sequence, we are able to show that the approximate entropy of the string distinguishes between common network ensembles; (4) we then consider visibility graphs, since these objects are associated with dynamical systems and therefore present a natural time ordering. We show that approximate entropy of those visibility graphs allows us to distinguish between series generated by different types of process.

The remainder of the paper is organized as follows. In Sec. II we recall the definition and main properties of approximate entropy. In Sec. III we study how to extend such notion to the context of networks, defining a network-based approximate entropy. Given this measure, we study both the case of static networks (Sec. IV), where the measure is purely structural, and the case of growing networks (Sec. V), where the measure acquires a more dynamical meaning. This latter situation is studied within the context of visibility graphs $[19,20]$. The measure is finally tested in Sec. VI with real data. By considering networks constructed from data obtained in the context of cancer statistics, we probe the capability of the measure to distinguish among different cancer phenotypes. Finally, we present a discussion in Sec. VII. The main open question concerns the study of approximate entropies of parameters beyond the degrees. One may take a number of different approaches depending on the parameters considered. Sequences of combinatorial nature obtained from counting paths and sequences of algebraic nature, such as graph spectra, seem to be good candidates for this purpose. Concerning the latter idea, it is an open direction to determine whether approximate entropy has any role in characterizing matrix ensembles when applied to their spectra.

\section{APPROXIMATE ENTROPY}

We begin by recalling the notion of approximate entropy. Due to Pincus [18,21-23], its definition is based on ideas of Eckmann, Ruelle, and ultimately Kolmogorov-Sinai. Whereas originally defined for time series, when the series is drawn from an alphabet of finitely many symbols, it has a powerful combinatorial interpretation due to Rukhin [24]. Moreover, approximate entropy has in general a geometric interpretation given when comparing "densities" of the Takens embedding of the time series in dimensions $m$ and $m+1$. Indeed, let $m \in \mathbb{N}$, $r \in \mathbb{R}_{>0}, u=(u(t))_{t=1}^{N}$ a time series of $N$ points and consider the $m$ th Takens embedding delay map $x(t)=(u(t), u(t+$
1), $\ldots, u(t+m-1))$, with image $X_{m}=\{x(1), \ldots, x(n)\} \subset \mathbb{R}^{m}$, where $n=N-(m-1)$. Recall that if $u$ arises from a dynamical system with a strange attractor of box dimension $d$ then, when $m>2 d$, the image $X_{m}$ "reconstructs" the strange attractor in an appropriate sense. We write $x_{i}(t)$ for the $i$ th component of $x \in \mathbb{R}^{m}$ and let $\|\cdot\|_{\infty}$ be the usual $L^{\infty}$ norm on $\mathbb{R}^{m}$, i.e., $\|x\|_{\infty}=\max _{i}\left|x_{i}\right|$. By denoting

$$
\Phi_{m}(r)=-\frac{1}{\left|X_{m}\right|} \sum_{x \in X_{m}} \ln \left(\frac{\left|\left\{y \in X_{m}:\|x-y\|_{\infty} \leqslant r\right\}\right|}{\left|X_{m}\right|}\right),
$$

the approximate entropy of the $N$ data points $u$ is defined as

$$
\operatorname{ApEn}(m, r, N)=\Phi_{m+1}(r)-\Phi_{m}(r)
$$

with the convention that $\operatorname{ApEn}(0, r, N)=\Phi_{1}(r)$ and $X_{0}=\{\}$. The upshot of this is that small values of ApEn imply strong regularity (or persistence), while large values amount to considerable irregularity in the time series $u$. From a more concrete perspective, approximate entropy can be interpreted as a measure of how far a sequence is from having a clear repetitive structure. The geometric picture is completed by observing that the Eckmann-Ruelle entropy is indeed recovered in the small $r$, large $m$ limit:

$$
\lim _{r \rightarrow 0} \lim _{m \rightarrow \infty} \lim _{N \rightarrow \infty} \operatorname{ApEn}(m, r, N) .
$$

Among the practical uses of approximate entropy, for example when studying time-series data of financial markets [25] or heart EEG data [26,27], the literature often uses $m=1$ or 2, together with $r$ proportional to the standard deviation.

The combinatorial picture gives the key insight for estimating the limiting distribution of approximate entropy and also identifying sequences of extremal approximate entropy. Indeed, let $u \in\{0,1, \ldots, S-1\}^{N}$ be a sequence of length $N$ on $S$ symbols (here we are taking $0<r<1$ ). Let $v_{i_{1}, \ldots, i_{m}}$ be the frequency with which the block $\left(i_{1}, \ldots, i_{m}\right) \in\{0,1, \ldots, S-1\}^{m}$ occurs in $\tilde{u}=\left(u_{1}, \ldots, u_{N}, u_{1}, \ldots, u_{m-1}\right)$. This amounts to the frequency of the block in $u$ arranged around in a circle. As before, set

$$
\widetilde{\Phi}_{m}=-\sum_{I \in\{0,1, \ldots, S-1\}^{m}} v(I) \ln v(I) .
$$

The modified approximate entropy is

$$
\widetilde{\operatorname{ApEn}}(m):=\widetilde{\Phi}_{m+1}-\widetilde{\Phi}_{m}
$$

so that its computation amounts purely to counting the relative frequencies associated with every possible block of length $m$ occurring in the sequence. This allowed Rukhin to get analytic proofs of the distribution of ApEn: For fixed embedding dimension $m$, we have then

$$
2 N(\ln S-\widetilde{\operatorname{ApEn}}(m)) \rightarrow \chi^{2}\left(S^{m+1}-S^{m}\right),
$$

where $\chi^{2} r$ denotes the so chi-squared distribution with $r$ degrees of freedom. Thus, the same behavior follows for $\operatorname{ApEn}(m)$ because

$$
N(\operatorname{ApEn}(m)-\widetilde{\operatorname{ApEn}}(m))=O_{\mathbb{P}}\left(\frac{1}{N}\right) .
$$




\section{APPROXIMATE ENTROPIES OF A NETWORK}

The degree sequence of a (finite, unweighted, and undirected) network $G$ with nodes labeled $\{1, \ldots, N\}$ is $d=$ $\left(d_{1}, \ldots, d_{N}\right)$ where $d_{1} \geqslant \cdots \geqslant d_{N}$ and $d_{i}=\operatorname{deg}(i)$ [3]. Note at this point that not all decreasing sequences of integers are degree sequences of networks. The best known criterion was given by Erdös and Gallai [28]: $d_{1} \geqslant \cdots \geqslant d_{N}$ is the degree sequence of a network with $N$ nodes if and only if $\sum d_{i}$ is even and $\sum_{i=1}^{k} d_{i} \leqslant k(k-1)+\sum_{i=k+1}^{N} \min \left(d_{i}, k\right)$ holds for every $1 \leqslant k \leqslant N$. What this really says is that the trivial bounds for the partial sums $d_{1}+\cdots+d_{k}$ of the first $k$ largest degrees, obtained by combining the contributions of $k(k-1)$ for the links between those first $k$ and $\min \left(d_{k+1}, k\right)+\cdots+\min \left(d_{N}, k\right)$ for the rest, is in fact optimal. In other words, whenever the condition is satisfied for every $k$, there exists a network with such a degree sequence. A constructive proof of this fact was given by Tripathi, Venugopalan, and West in [29]. In fact, Tripathi and Vijay [28] observed that it is enough to check the condition for $k=n$ and every $k$ such that $d_{k}>d_{k+1}$.

Good asymptotic bounds for the number of degree sequences of networks on $N$ nodes were given by Burns [30]; in this reference it was shown that the existence of constants $C_{0}$ and $C_{1}$ such that the number of degree sequences of graphs on $N$ vertices, $\Delta_{N}$, satisfies $4^{N} / C_{0} N \leqslant \Delta_{N} \leqslant 4^{N} /(\ln N)^{C_{1}} \sqrt{N}$. It is worth noting however that among the sensible candidates for degree sequences, i.e., among monotone decreasing sequences of the appropriate length, the proportion of these that are degree sequences tends to zero, a fact conjectured by Wilf and proven by Pittel [31].

From now on, let $d_{1} \geqslant \cdots \geqslant d_{N}$ be the degree sequence of a finite network with distinct values $D_{1}>\cdots>D_{s}$ such that $D_{i}$ occurs $n_{i}$ times (so $N=n_{1}+\cdots+n_{s}$ ). How best to assign an approximate entropy to a degree sequence? We consider three options in turn:

(1) The simplest thing to do is just to compute the approximate entropy of this monotone sequence viewed as a time series, due to a lack of a natural ordering of nodes.

(2) More sophisticated is to assign some combinatorial description of the degree sequence, with potentially interesting entropic properties.

(3) For networks with a natural ordering to their vertices, we can compute approximate entropy of such an ordering. For example, a network that has grown by a process of sequential node addition has a natural ordering on the nodes according to when they were added. An example of this is the standard Barabási-Albert model of preferential attachment [33].

\section{A. The degree sequence as a monotone time series}

Option (1) does not seem to coincide with intuition of what an entropic sequence should be: Indeed if $m$ and $m+1$ divide $N$ then in the small $r$ limit, the behavior captures more the dimension than the disorder in the sequence. This feature is highlighted by the following observation: Suppose that $D_{i}-D_{i+1}>r$; then $\operatorname{ApEn}(m, r, N: m$ and $m+1$ divide $N)$ is maximal when $s=N / m$ and $n_{1}=\cdots=n_{s}=m$ and minimal when $n_{1}=\cdots=n_{s}=m+1$. Let us sketch how to show this statement. We may take $0<r<1$ and suppose the $D_{i}$ to be integers. Optimizing $\operatorname{ApEn}(m, r, N)=\Phi_{m+1}-\Phi_{m}$ for a degree sequence $d$ amounts to optimizing the ratio $R$ of geometric means of the numbers of points in $X_{m}$ of $\|\cdot\|_{\infty}$ distance $\leqslant r$ for each $x \in X_{m}$. Hence,

$$
R=\frac{\left(\prod_{x \in X_{m}}\left|\left\{y \in X_{m}:\|x-y\|_{\infty} \leqslant r\right\}\right|\right)^{1 /\left|X_{m}\right|}}{\left(\prod_{x \in X_{m+1}}\left|\left\{y \in X_{m+1}:\|x-y\|_{\infty} \leqslant r\right\}\right|\right)^{1 /\left|X_{m+1}\right|}} .
$$

In this case, $0<r<1$ implies that we need only optimize

$$
R=\frac{\left(\prod_{i=1}^{s} \Delta_{i}(m)^{\Delta_{i}(m)}\right)^{1 /\left|X_{m}\right|}}{\left(\prod_{i=1}^{s} \Delta_{i}(m+1)^{\Delta_{i}(m+1)}\right)^{1 /\left|X_{m+1}\right|}},
$$

i.e.,

$$
R=\prod_{i=1}^{s} \frac{\Delta_{i}(m)^{\Delta_{i}(m) /\left|X_{m}\right|}}{\Delta_{i}(m+1)^{\Delta_{i}(m+1) /\left|X_{m+1}\right|}} .
$$

Here $\Delta_{i}(m):=\max \left(1, n_{i}-(m-1)\right)$ such that $n_{1}+\cdots+$ $n_{s}=N$ and $1 \leqslant s \leqslant N$. We are also free to sort the $n_{i}$ such that $n_{1} \geqslant \cdots \geqslant n_{s}$. Now, suppose that there are $j \leqslant s$ separate $n_{i}$ such that $\Delta_{i}(m)=n_{i}-(m-1)>1$ and $k \leqslant j$ distinct $n_{i}$ such that $\Delta_{i}(m+1)=n_{i}-m>1$. Set $r_{i}=n_{i}-m$ then $r_{1} \geqslant \cdots \geqslant r_{j} \geqslant 3>2=r_{j+1}=\cdots=$ $r_{k}>1 \geqslant r_{k+1} \geqslant \cdots r_{s}$. The ratio $R$ now reads

$$
R=\prod_{i=1}^{j} \frac{r_{i}^{r_{i} /\left|X_{m}\right|}}{\left(r_{i}-1\right)^{\left(r_{i}-1\right) /\left|X_{m+1}\right|}} \prod_{i=j+1}^{k} 4^{1 /\left|X_{m}\right|} .
$$

The statement follows by writing out the behavior of $x^{x / k} /(x-$ $1)^{(x-1) /(k+1)}$ for $k$ an integer at least 2 .

Thus this gives only an idea of proximity to such nonintuitively entropic sequences. For example, with $0<r<1$ and $m=0$, the approximate entropy is maximal for the sequence $(N-1, N-2, \ldots,\lceil N / 2\rceil,\lceil N / 2\rceil, \ldots, 2,1)$; if $m=1$ and $N=9$, the maximum is realized by the sequence $(8,8,7,7,6,6,4,4,4)$. A graph is regular if all its vertices have the same degree. The frequency partition of a graph is a partition of its vertices grouped by their degrees. Such a notion is graph invariant, but intuitively there are many non-isomorphic graphs with the same frequency partition. It is known that every partition is a frequency partition of some graph, with the exception of $(1,1, \ldots, 1)$ (see [32]). A graph has a regular frequency partition if each block of the partition is of the same size. In general, if $m=1$, the graphs that realize the maximum have degree sequence $(N-1, N-1, N-2, N-$ $2, \ldots, N-(N+1) / 2, N-(N+1) / 2,(N-1) / 2)$ when $N$ is odd and $(N-1, N-1, N-2, N-2, \ldots, N / 2, N / 2)$ when $N$ is even (we take $N \geqslant 4$ ).

\section{B. The slide of a degree sequence}

Let us now explore option (2). Given a degree sequence $d=\left(d_{1}, \ldots, d_{N}\right)$, for each $i=1, \ldots, N-1$ write down 0 if $d_{i}=d_{i+1}$ and otherwise write a string of $d_{i}-d_{i+1} 1$ 's if $d_{i}>d_{i+1}$. We denote this sequence by slide $(d)$. For a network $G$ with degree sequence $d$, write $\operatorname{side}(G)$. Note that for a network $G$ on $N$ nodes, $N-1 \leqslant$ No. of $\operatorname{slide}(G) \leqslant 2(N-2)$ with the minimum length attained by (for example) regular networks and the maximum attained by stars (i.e., complete bipartite networks with the singlet as a class of the bipartition). Thus a degree sequence with $s$ distinct degrees is associated 
with a binary sequence of $N-s$ zeros and $d_{1}-d_{N}$ ones and the collection of associated sequences of networks with $N$ nodes is a certain subset of binary sequences with up to $N$ zeros and up to $N-1$ ones. For example, the degree sequence $(4,3,3,3,1)$ is encoded as $(1,0,0,1,1)$. The associated binary code has a simple interpretation in terms of "sliding" down the degree sequence: 0 means "go horizontally right" and 1 means "continue going down." Note that not every binary sequence arises as the slide of some network. For example, there is no network $G$ with $\operatorname{slide}(G)=001$ : Indeed such a degree sequence must be $d_{1}=d_{2}=d_{3}=d_{4}+1$ and none of the possibilities $(3,3,3,2),(2,2,2,1)$, or $(1,1,1,0)$ are graphical because they all have odd sum. Obviously the slide map is not injective and further, networks of different numbers of nodes can have the same slides. The approximate entropy of binary sequences was studied by Pincus [23] in the context of developing properties of normal numbers. The notion of approximate entropy allows us to compare binary sequences. The language used by Pincus to do so is as follows: A binary sequence $u \in\{0,1\}^{N}$ of length $N$ is called " $(m, N)$ random" if $\operatorname{ApEn}(m, N)(u)$ is maximal among all binary sequences of the same length. Let $m^{*}(N)$ be the largest integer such that $2^{2^{m^{*}(N)}} \leqslant N$, and call a binary sequence $u$ " $N$ random" if for $m=0,1,2, \ldots, m^{*}(N)$ it is $(m, N)$ random. Finally, we can compare two binary sequences $u$ and $v$ of the same length $N$ and say that $u$ is more $N$ random than $v$ if $\operatorname{ApEn}(m, N)(u) \geqslant$ $\operatorname{ApEn}(m, N)(v)$ holds for all $m$ such that $1 \leqslant m \leqslant m^{*}(N)$.

A characterization of binary sequences of the largest approximate entropy was also given by Pincus: For $N \geqslant 5$, the $N$-random binary sequences amount to equivalence classes of sequences of length $N$ of a partially exchangeable process in which "approximate stability of frequencies holds," in the sense that

$$
\left|\frac{\text { No. of }\left\{\left(a_{0}, \ldots, a_{m}\right) \text { blocks in the sequence }\right\}}{N-m}-\frac{1}{2^{m+1}}\right|
$$

is as small as possible for each block type $\left(a_{0}, \ldots, a_{m}\right) \in$ $\{0,1\}^{m+1}$ and for every $0 \leqslant m \leqslant m^{*}(N)$. That such sequences are asymptotically of large approximate entropy can be seen immediately from Rukhin's characterization. What this says is that the binary sequences of maximal approximate entropy amount to optimal "truncations" of normal numbers written in base 2 .

If asymptotically all binary sequences arose as the slides of some degree sequence, while the proportion of binary sequences of length $n$ that are $n$ random tends to 0 , we could expect networks whose slides were arbitrarily nearby (it is unknown for which $n$ there exist networks with $n$-random slides). In particular, we conjecture the following statement: The probability that a uniformly chosen slide of length $n$ is graphical tends to 1 as $n \rightarrow \infty$. Even if the outcome of this is largely a curiosity, it is recorded to assert the existence of sufficiently many slides to guarantee a variety of distinct values of the approximate entropy. In other words, this would show that the notion asymptotically exhibits a good range. A potentially fruitful approach to a proof proceeds in three steps: First, one may consider Pittel's approach for proving the Wilf conjecture. The approach refines the insight of Erdös-Richmond in associating an integral estimate of the probability of surviving the Nash-Williams graphicality condition. Subsequently, one should combine the Kolmogorov 0-1 law with a sample family of networks whose slides make an asymptotically nonzero contribution. An alternative is to try show that with high probability, one can construct a network (perhaps via the arguments of [29]) exploiting the "slack" gifted by the considerable nonuniqueness of slides. We have computed the first few terms of the sequence $s_{2}, s_{3}, \ldots, s_{6}$. The respective values are $3 / 4,3 / 8,13 / 16,20 / 32,58 / 64$. We are now ready to define the slide entropy of a network:

Definition. If $G$ is a network with $N$ nodes then the slide entropy of $G$ in dimension $m$ is

$$
\operatorname{Slide} \operatorname{ApEn}(G, m)=\operatorname{ApEn}(\operatorname{slide}(G), m, r)
$$

for any $0<r<1$. This notion is the topic of the next section.

\section{APPROXIMATE ENTROPY OF SLIDE SEQUENCES: APPLICATION TO STATIC COMPLEX NETWORKS}

Scale-free networks are of interest due to their abundance in nature and technology [2,3,33]. Computationally, estimates of their behavior as complex networks are often made by treating their degree sequences as random variables sampled from a scale-free distribution $\pi(x)=(\gamma-1) x^{-\gamma}$ (for real $x>0$ ). A natural construction via growth through preferential attachment was popularized by Barabási and Albert [33]. The probability distributions beyond the degree distribution this yields on networks of $N$ nodes (for each $N$ ) is quite distinct from simply sampling a scale-free distribution and trying to assemble a network from that, the so-called configuration model [2]. Scale-free networks are often compared with an older and very well studied notion of random network, introduced by Erdös and Rényi; these are constructed by adding each edge with a fixed probability $p$. For $N$ nodes, with $\lambda=N p$, their degree distribution is asymptotically Poisson, $\pi(x)=\lambda^{k} e^{-\lambda} / k$ ! $(x>0)$. Also of interest are random networks with exponential degree distributions, which naturally arise as the renormalization group fixed point of visibility graphs associated with random uncorrelated time series, with $\pi(x)=\lambda e^{-\lambda x}(x>0)[20,34]$.

The approximate entropy of a generic slide with $d_{1}-d_{N}$ ones and $N-s$ zeros can be computed by considering the Markov chain with state space $X=\{0,1\}$ and transition matrix $P$ given by $P_{00}=P_{10}=p$ and $P_{11}=P_{01}=q=1-p$ [thus $\pi(0)=p$ and $\pi(1)=q]$. We recall the following result of Pincus. For a first-order stationary Markov chain with discrete state space $X \subset \mathbb{N}$, transition probabilities $P_{x y}=$ $\mathbb{P}$ (travel from $x$ to $y$ ), and stationary distribution $\pi$ with $r<$ $\min _{x \neq y \in X}|x-y|$, then almost surely for every $m$

$$
\lim _{N \rightarrow \infty} \operatorname{ApEn}(m, r, N)=-\sum_{x, y \in X} \pi(x) P_{x y} \ln \left(P_{x y}\right) .
$$

It follows that a good approximation is often given (asymptotically and independently of $m$ ) by

$$
\operatorname{SlideApEn}(G, m) \approx H\left(\frac{N-s}{N-s+d_{1}-d_{N}}\right),
$$

where as usual $H(p)=-[p \ln p+(1-p) \ln (1-p)]$. However for a scale-free network on $N$ nodes with degree distribution sampled from a truncated scale-free distribution $\tilde{\pi}(k) \sim k^{-\gamma}$ for $k \in\{1, \ldots, N-1\}$. In the large $\gamma$ limit the 

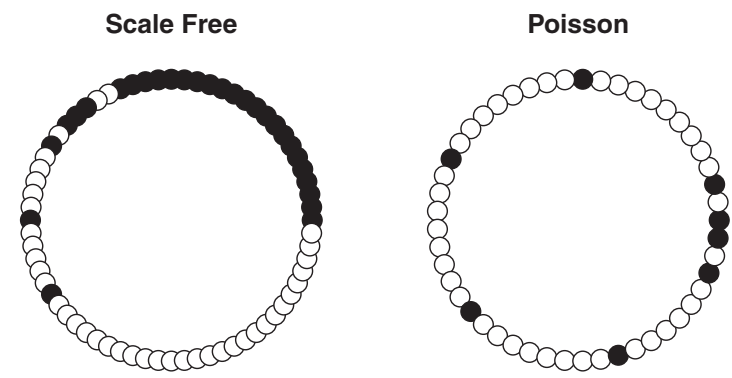

FIG. 1. Circularly arranged slides of random scale free and Poisson networks of $N=50$ nodes illustrating typical structure. Note that while $N=50$ is perhaps far too small to meaningfully refer to the distribution as scale free the figure is intended only to be illustrative of the general appearance of the slides.

network is dominated by degree 1 nodes as we can expect to have at least $N k^{-\gamma} / \zeta(\gamma)$ nodes of degree $k$ which tends to zero for all $k$ except $k=1$. This creates large regions of zeros in their slides. Assuming generic behavior in the region of the slide not accounting for the degree 1 nodes, the ApEn estimate of Rukhin suggests intuitively that the slide entropy should be roughly monotone decreasing in $\gamma$ for each fixed $N$. This intuition can be seen numerically in Fig. 1. Estimating $d_{1}-d_{N}$ can be done by using the following elementary result. Let $X_{1}, \ldots, X_{N}$ be drawn from a probability distribution $\pi$ on $\mathbb{R}$ with cumulative density $\Pi$. Then $E_{m}:=\mathbb{E}\left(m\right.$ th largest $\left.X_{i}\right)$ is given by

$$
\int_{\mathbb{R}} x \pi(x) \frac{N !}{(N-m) !(m-1) !} \Pi(x)^{m-1}[1-\Pi(x)]^{N-m} d x .
$$

As usual, here we denote by $\mathbb{E}$ the expectation operator. If we are drawing the degrees $\operatorname{deg}(v)$ of nodes $v \in\{1, \ldots, N\}$ then $\mathbb{E}\left(d_{1}-d_{N}\right)=E_{1}-E_{N}$ can be computed for the scale-free degree distribution (we have $E_{N}=1$ almost surely) given approximately (following Ghoshal-Barabási [35]) by

$$
E_{1} \approx(N-1)^{1 /(\gamma-1)} \Gamma\left(\frac{\gamma-2}{\gamma-1}\right) .
$$

The approximation for $E_{1}$ is good for large $N$, but performs poorly near $\gamma=2$ due to the pole at 0 . To estimate the expected number of distinct degrees $s$, we associate with the continuous probability distribution $\pi$ on $\mathbb{R}$ a distribution $\bar{\pi}$ on $\mathbb{N}$. An elementary argument says that the expected number of distinct values of a random sample of size $N$ from the distribution $\bar{\pi}$ is

$$
s(N)=\sum_{n \in \mathbb{N}}\left(1-[1-\bar{\pi}(n)]^{N}\right) .
$$

In this way, for a sample of $N$ random variables from a scale-free distribution $\bar{\pi}(n):=n^{-\gamma} / \zeta(\gamma)$ and upon comparing with the integral, we approximate $s(N)$ by the following hypergeometric function:

$$
s(N) \approx{ }_{2} F_{1}\left(-\frac{1}{\gamma},-N ; \frac{\gamma-1}{\gamma} ; \frac{1}{\zeta(\gamma)}\right) .
$$

This provides an analytic expression for the entropy of a generic slide of the same $0-1$ distribution, but is a considerable overestimate for real scale-free slides. This is a bad approximation but the previous computations illustrate

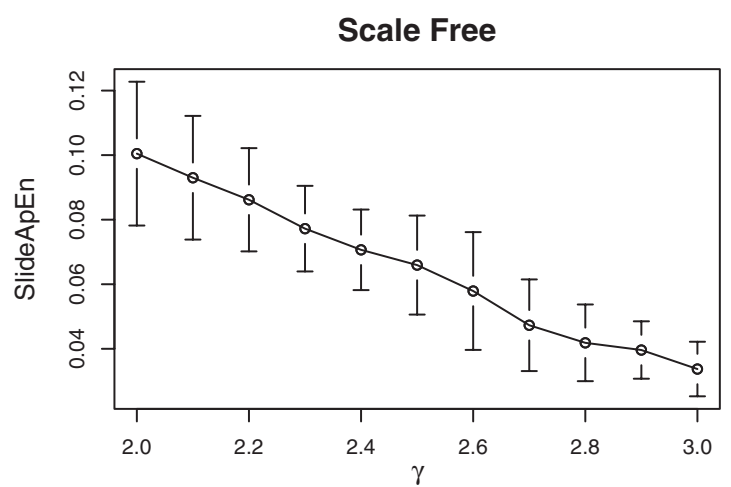

Generic "Scale Free"

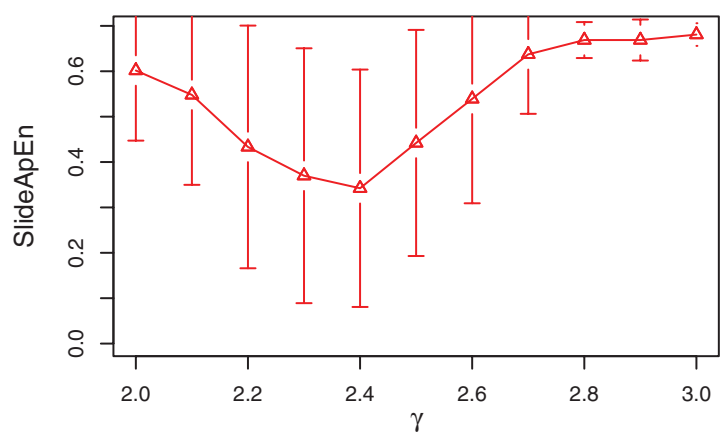

Poisson

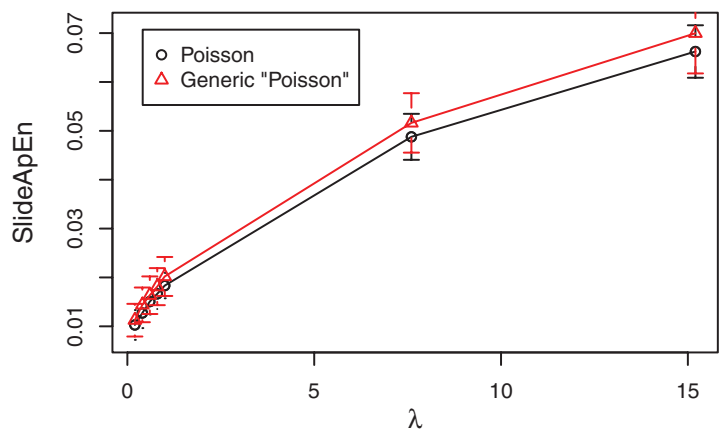

FIG. 2. (Color online) Slide entropies for scale-free and Poisson (Erdös-Rényi) networks of $N=2000$ nodes. Generic values given are those of random slide entropies of slides with the same distribution of 0 's and 1's (corresponding to the analytic approximation for $\gamma \gg 2$ and for Poisson sequences). Error bars indicate two standard deviations obtained from simulations.

the approach for obtaining good analytics in other cases. We similarly obtain generic estimates for exponential degree sequences by computing $E_{1}-E_{N}=\frac{1}{\lambda} \sum_{i=1}^{N-1} \frac{1}{i} \sim \ln (N-$ $1)+O\left(\frac{1}{N}\right)$ and $s(N) \approx \int_{1}^{\infty}\left[1-\left(1-\lambda e^{-\lambda x}\right)^{N}\right] d x$. For Poisson networks, we find numerically that the generic estimate provides a good approximation. It is interesting to ask which probability distributions $\bar{\pi}$ on $\mathbb{N}$ tend to give rise to networks of the largest and smallest slide entropies. Among such distributions on $N$ nodes, we expect that the uniform distribution on $\{0,1, \ldots, N-1\}$ is of the greatest typical slide entropy. Note that the point distribution on any fixed $k \in\{0,1, \ldots, N-1\}$, such that the networks desired exist, always gives rise to 0 slide entropy networks (see Fig. 2).

To conclude this section, we note the heuristic difference between using the approximate entropic measure proposed 
and existing alternatives including the variance and Shannon entropy of the degree distribution viewed as a probability distribution on the vertices. Indeed, approximate (slide) entropy works by measuring how far away the degree sequence is from decreasing in a predictable and repetitive manner, while the variance simply measures how "spread out" the different degrees are. Further, the Shannon entropy measures more how distinct the overall shape of the distribution is from being the uniform distribution on a finite set of points. As such the variance and Shannon entropy do not see the subtleties of the ordering of the sequence. Slide entropy also encodes entirely different information from the Gibbs entropy considered by Bianconi [11,12] which effectively computes the numbers of networks with a given degree distribution.

\section{APPROXIMATE ENTROPY OF GROWING NETWORKS: HORIZONTAL VISIBILITY GRAPHS}

Within nonlinear time series analysis, the so-called visibility algorithms [19,20,34,36,37] are a family of methods that directly map a given time series of $N$ data into a network of $N$ vertices (a so-called visibility graph), where the edge set is constructed according to specific geometric criteria to be applied among the data set. In previous works it has been shown that the associated visibility graph of a time series with a given information is conserved or inherited in the topology of the associated visibility graph, including nontrivial structures such as chaotic or fractal dynamics. To cite a few, within the so-called visibility algorithm approach [19,36], series extracted from a fractional Brownian motion with Hurst exponent $H$ map into scale-free visibility graphs with degree distribution $P(k) \sim k^{-\gamma}$, where the linear relation $\gamma=3-2 H$ quantitatively relates the structure of the dynamical process $(H)$ with the topology of the associated graph $(\gamma)$. Within an alternative approach coined as the horizontal visibility algorithm [20,38], it was shown [37] that horizontal visibility graphs distinguish between correlated stochastic, uncorrelated, and chaotic processes, and in each of these cases the visibility graph has exponential degree distribution $P(k) \sim e^{-\lambda k}$ with the value of $\lambda$ characterizing the particular process. Recently, it has been also suggested that the Shannon entropy over the degree distribution of a horizontal visibility graph is a first-order approximation to the Kolmogorov-Sinai entropy of the associated dynamical system [34].

As an initial observation, note that the visibility graph associated with a given time series conserves, by construction, the temporal ordering of the data, i.e., temporal correlations among the data. This is due to the fact that in the mapping algorithm, each datum $x_{i}$ maps into a labeled vertex $n_{i}$, that is to say, a natural ordering of the vertex set emerges, respecting the temporal correlations in the series. The implications are twofold: (i) Within (horizontal) visibility graphs one has a natural ordering of the degree sequence, which allows us to unambiguously calculate the approximate entropy of such series, and (ii) since that ordering is related to the temporal correlations of the associated series, the approximate entropy of a visibility graph may provide a measure of the associated series complexity; that is, it becomes a measure directly related to the original one introduced by Pincus in the framework of dynamical systems. In other words, whereas our previously defined, network-based slide entropy accounts for the heterogeneity of the degree sequence itself (with no dynamical or temporal information whatsoever), in the context of visibility graphs this network-based measure is indeed capturing some dynamical information. Notice also that since each datum in a time series is associated with a labeled vertex in the graph, one can view a visibility graph as a dynamically growing network: As time evolves, the dynamical process generates a trajectory (time series) whose associated visibility graph grows. The approximate entropy of its degree sequence accounts for the information stored in the network growing process.

In order to test the aforementioned conjectures, we will address, within the so-called horizontal visibility algorithm, three types of time series whose associated approximate entropy, associated with the amount of information needed to unravel the underlying dynamics, is qualitatively different: periodic series (i.e., regular dynamics with pointlike attractor measure), chaotic series (deterministic dynamics with finite attractor measure), and white noise (stochastic dynamics with infinite attractor measure). We proceed as follows: Let $\left\{x_{t}\right\}_{t=1, \ldots, N}$ be a real-valued time series of $N$ data. The horizontal visibility algorithm assigns each datum of the series to a vertex in the horizontal visibility graph $(\mathrm{HVg})$. Then, two vertices $i$ and $j$ in the graph are connected if one can draw a horizontal line in the time series joining $x_{i}$ and $x_{j}$ that does not intersect any intermediate data height. Hence, $i$ and $j$ are two connected nodes if the following geometrical criterion is fulfilled within the time series:

$$
x_{i}, x_{j}>x_{n}, \forall n \mid i<n<j .
$$

The generated $\mathrm{HVg}$ has a degree sequence of the kind $\left\{k_{1}, k_{2}, \ldots, k_{N}\right\}$, where $k_{i}$ is the degree of vertex $i$, that is to say, associated to datum $x_{i}$ in the original series (as opposed the definition in Sec. III, note that this degree sequence is not monotonically decreasing since it has a natural ordering already explained above). We finally calculate our networkbased ApEn. Results for periodic, chaotic, and noisy series, for specific values of the ApEn parameters, are summarized in Table I and in Fig. 3. In this respect, we can highlight the following comments:

TABLE I. Values of ApEn for concrete parameters $m=2, r=2$, and size $N=2^{14}$ of the $\mathrm{HVg}$ associated with three types of time series: (i) a periodic series of period 2 (deterministic dynamics with an underlying attractor of zero measure), (ii) a chaotic series extracted from the fully chaotic logistic map $x_{t+1}=4 x_{t}\left(1-x_{t}\right)$ (deterministic dynamics with an underlying attractor of finite measure), and (iii) a series of uncorrelated random variables extracted from a uniform distribution $U[0,1]$ (stochastic dynamics, i.e., dynamics with a hypothetical infinite-dimensional attractor). The approximate entropy of the visibility graphs increases as a function of the associated series information.

\begin{tabular}{lc}
\hline Series Description & $\operatorname{ApEn}\left(2,2,2^{14}\right)$ \\
\hline Periodic series $(T=2)$ & 0.001 \\
Chaotic series (logistic map, $\mu=4)$ & 0.47 \\
$U[0,1]$ uncorrelated & 0.62 \\
\hline
\end{tabular}



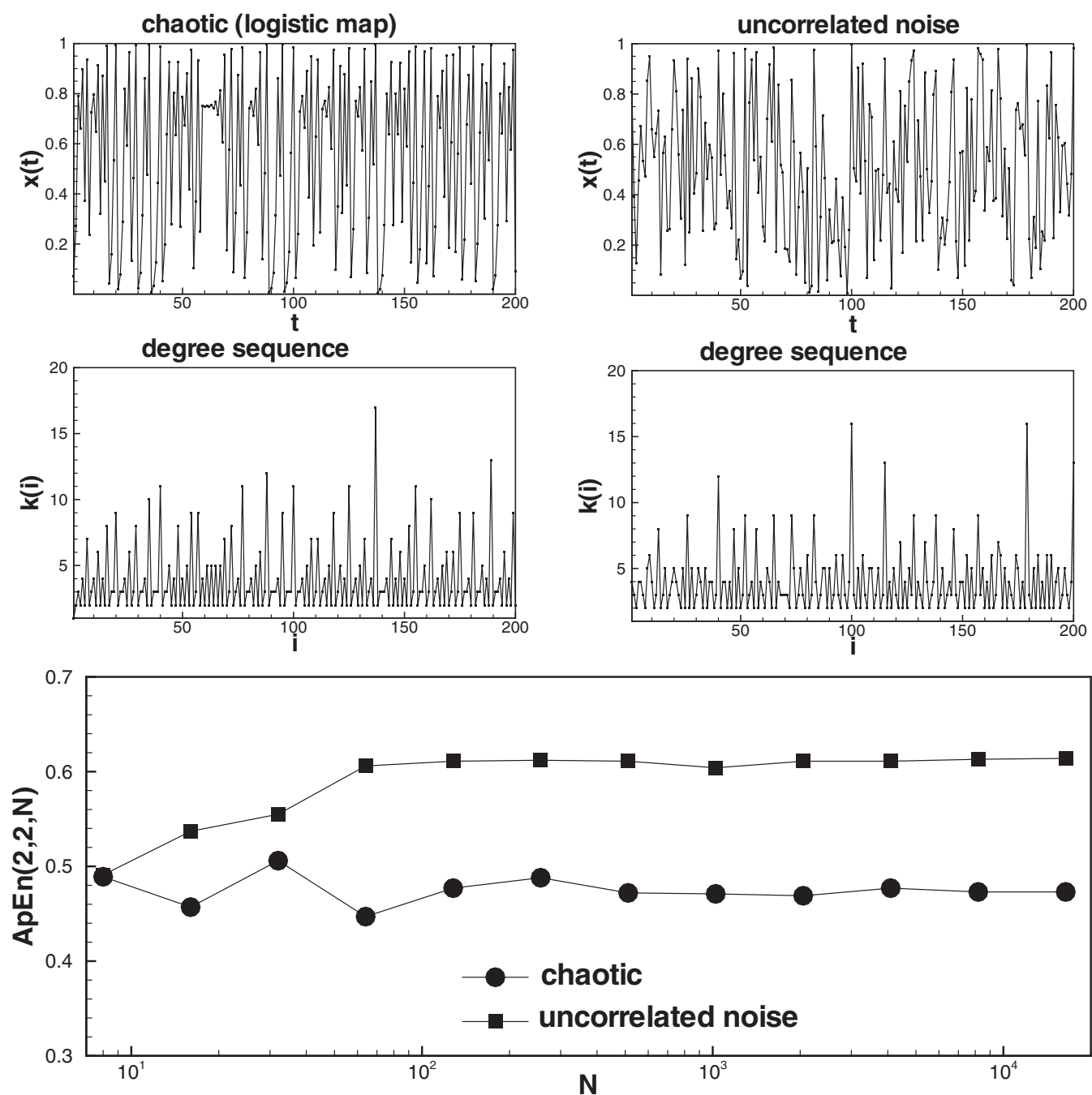

FIG. 3. Top: Sample series extracted from (left) the fully chaotic logistic map $x_{t+1}=4 x_{t}\left(1-x_{t}\right)$ and (right) uncorrelated random variables from $U[0,1]$. Below, we show a sample of the degree sequences of their associated HVg's. Bottom: Values of $\operatorname{ApEn}(2,2, N)$ of each $\mathrm{HVg}$, as a function of the series size $N$. Note that the time is in logarithmic scales. The values for the graph associated with the noisy process are larger than those associated with the chaotic process, in concordance with the entropy associated with the underlying dynamical process.

(1) HVg associated with periodic series: By construction, these networks have a very homogeneous structure [20], which can indeed by seen as a concatenation of a network motif, the structure of this network motif being intimately related to the series periodicity. Accordingly, their ApEn is small, having a vanishing value for large embedding dimension $m$. If we make use of small values of $m$, the ApEn statistic can be used to distinguish several degrees of periodicity, associated with the heterogeneity of the visibility network root motif.

(2) Visibility graphs associated with random uncorrelated white noise: White noise is a maximally entropic signal according to any well-defined information theoretic measure. In a previous work [34], it was shown that the Shannon entropy over the degree distribution of an $\mathrm{HVg}$ is indeed maximized for uncorrelated white noise. Here, unlike periodic series, results are close to convergence for $m=2$. For a given series size $N$, noise yields the maximal ApEn, but this value increases with the series size $N$, as it should.
(3) Visibility graphs associated with chaotic maps: The ApEn of the associated networks reach a nonzero value, reminiscent of the underlying attractor of the dynamics. Convergence is reached for $m=2$ and, unlike noise, convergence as a function of series size is also reached here, as it should be. This preliminary analysis suggests that the network structure captures the inherent complexity of the associated time series. This supports the well-posedness of the visibility graphs as a tool for time-series analysis. Based on this conclusion, we can also point out that the network theoretical variation of the approximate entropy statistic can effectively distinguish different network structures according to their associated "complexity."

Mixed statistic. So far, two alternative ways have been considered in order to define the sequence over which the approximate entropy is computed, namely (i) networks without a predefined time arrow-computation is performed over the slide of the (monotonically decreasing) degree sequence - and (ii) growing networks — computation is 
performed over the (time ordered) degree sequence. A mixed approach consists of computing ApEn over the slide of a time-ordered degree sequence. We consider an example of this next.

\section{APPROXIMATE ENTROPY AND BIOLOGICAL NETWORKS: AN APPLICATION TO CANCER GENOMICS}

Finally, we focus on a potential application of these ideas to the field of cancer genomics. A key feature of cancer genomes is the abnormal copy number of genes. Since healthy cells are diploid they have 2 copies of each gene; however, in cancer cells, genes are deleted or may be present in multiple copies. Genes also have a natural ordering since they can be located to specific positions on the genome. Thus, for each tumor we can measure a copy-number profile along the genome. For technical reasons this is represented as a continuous valued variable (segmented data) [39], with neighboring genes more likely to have the same value. This copy-number profile varies along the genome, and can therefore be mapped as a time series, where genomic position plays the role of time. Thus, an $\mathrm{HVg}$ can be constructed for this genomic series of copy number values. We hypothesized that this $\mathrm{HVg}$ construction could encapsulate important information concerning the distribution and shape of the copy-number profiles of each individual tumor, a hypothesis that we test a posteriori by correlating the resulting entropy scores with known cancer phenotypes.

As a data set we considered the copy-number data of 171 breast cancer patients [39], for which three phenotypic categories were available: estrogen receptor status (ER), whether the patient's tumor metastasized or not (DM), and histological grade (3 levels represent levels of differentiation from normal healthy tissue). For each tumor we computed the slide and ordered entropies from the $\mathrm{HVg}$ graphs and asked whether these differed between phenotypes.

For brevity, we shall say that two phenotypes are distinguished by some associated quantity if the means of the quantity for each phenotype are statistically significantly different (say, around the 5\% level). Interestingly, we find increases in both entropies between ER-negative (0) and ER-positive (1) breast cancer, between the successive grades of the cancers, and in relation to distant metastasis (DM). In particular, the slide and ordered approximate entropy distinguish grade 1 breast cancer from grade 3 (Welch $t$ test with $p$ value 0.003 and 0.0004 , respectively). However, slide entropy better distinguishes distal metastasis ( $p$ value of 0.036 compared with 0.242 ) while ordered approximate entropy of the $\mathrm{HVg}$ better distinguishes ER status ( $p$ value of 0.005 compared with 0.057$)$. These results show that the ApEn and $\mathrm{HVg}$ construction can indeed capture interesting clinico-pathological features of cancer genomes. Our analysis is reported in Fig. 4.
ER

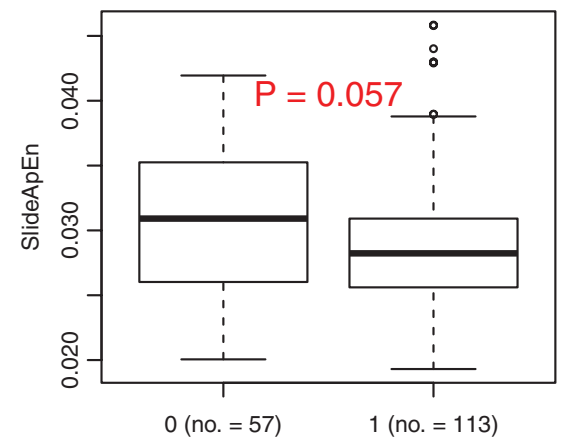

ER

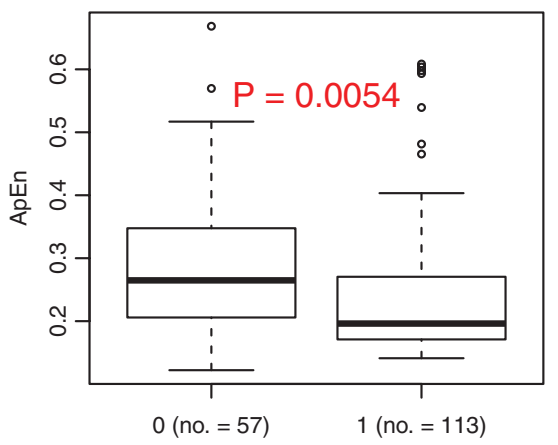

GRADE

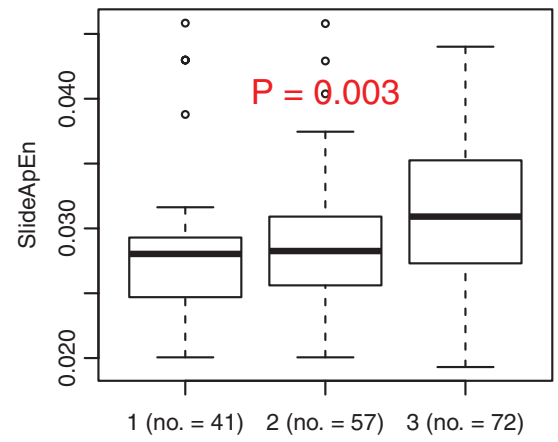

GRADE

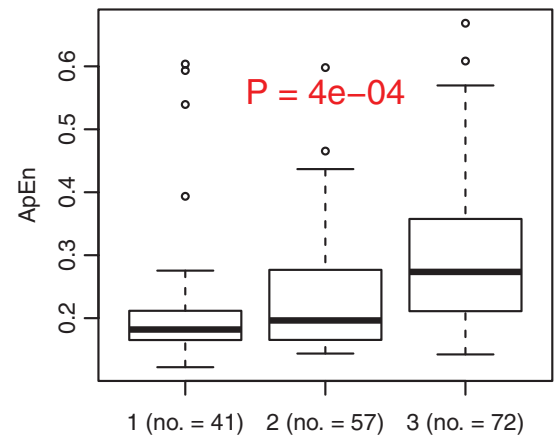

DM

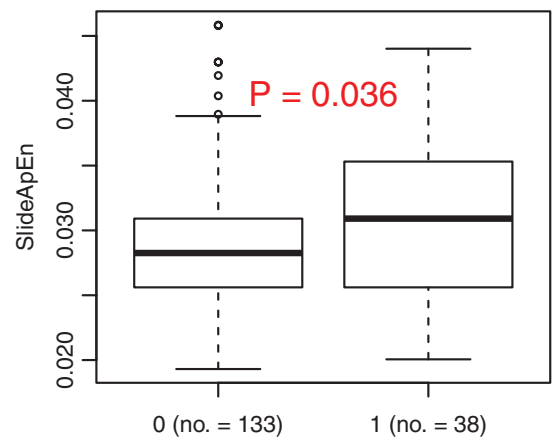

DM

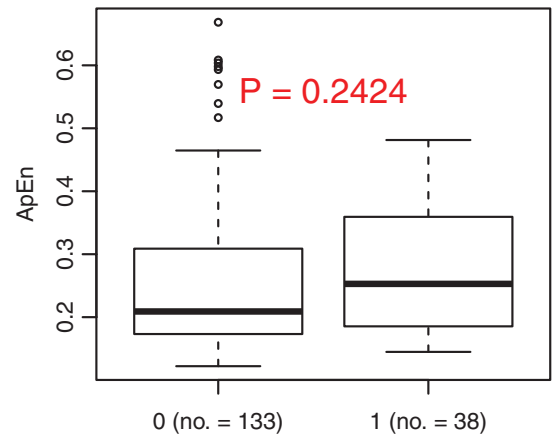

FIG. 4. (Color online) Slide entropy $(m=1)$ and ordered degree sequence entropy $(m=1$ and $r<1)$ of degree sequences of HVg's constructed from copy number data of 171 breast cancer patients split according to phenotype. ER denotes estrogen receptor status, indicating whether the cancer cells depend on estrogen for their growth, GRADE indicates the extent to which the cells have differentiated away from being normal cells, and DM denotes whether or not the patient has suffered distal metastasis (i.e., whether the cancer has spread). The bars represent $5 \%$ and $95 \%$ quantiles of the distribution. 


\section{CONCLUSIONS}

The original definition of approximate entropy quantifies the structure of a system's underlying phase space by looking at time-evolving trajectories over such phase space; hence it requires a time-ordered sequence. If the system under study is a network, its structure can still be studied with a use of approximate entropy, but it obviously requires some modifications or assumptions. If the network under study is generated by a dynamical process, then a natural time ordering can be defined over the vertex set, and ApEn is naturally extended to this domain: Such is the case of visibility graphs addressed in this work; further study of other dynamically growing networks, such as Barabási-Albert-like, is left for future research. Conversely, if the network under study is static, the natural extension of ApEn is not straightforward. In this case, we can still compute the approximate entropy of various networks' parameters, but this requires us to make some choices. In this work we have computed the approximate entropy of parameters related to the degree sequence. In the attempt of capturing only the relevant information, we have introduced the slide sequence of a network. We have shown that approximate entropy permits us to distinguish between the usual ensembles of Poisson and scale-free networks.

Moving from static to dynamical networks, we have focused on horizontal visibility graphs. Our findings suggest that the approximate entropy of these objects is intimately related to the amount of information generated by the underlying dynamical process. Indeed, we have given further evidence that just the degree sequence of an $\mathrm{HVg}$ has the power of discriminating between different dynamical processes. Finally, we have applied our statistic to a specific $\mathrm{HVg}$ generated from cancer genomic data, and have shown that this statistic can discriminate cancer phenotypes according to their histopathological characteristics and malignant potential. While we have previously shown that more aggressive breast cancers are characterized by an increased molecular entropy at the gene expression level [40], the results obtained in this manuscript demonstrate that more aggressive breast cancers (ER negative or high grade) are also characterized by a higher entropy at the genomic copy-number level. In this regard, an appealing feature of the entropy measures considered here is that they are sample specific, and thus may provide valuable insights into tumor taxonomy as shown recently using a different nonentropic set of measures in [41]. It will therefore be very interesting to explore and compare the entropy measures considered here to other proposed measures in the context of tumor classification and prediction of clinical outcome. It will also be interesting but challenging to observe analogues of phase transitions in statistics related to this type of data. We briefly conclude with two open problems. The first problem is about giving an interpretation to the approximate entropy as defined here; the second one is about identifying naturally ordered sequences associated with networks.

(1) What does the slide ApEn tell us about a network? If we take a series and measure the ApEn of its visibility graph, what information do we learn from the dynamical process that generated the series? There should be a neat correlation between the ApEn of the series and the one of the visibility graph. Such a correlation may be used to determine what kind of information about a series is not seen by the visibility graphs approach. The gain is to uncover the limits of methods for time series analysis based on visibility graphs.

(2) We have already mentioned that ApEn makes sense only when applied to an ordered list of numbers obtained from a network. The first intuitive choice was the degree sequence when arranged in the nonincreasing order. Clearly, this is not only the most natural choice, but it is also the easiest one. There are many potential generalizations based on different criteria. From a dynamical viewpoint, one could label the vertex set and then generate random walks over a given network. These are analogous to run trajectories over a dynamical system's phase space. ApEn is then computed over these trajectories, as was performed for the KS entropy in [17].

From a combinatorial viewpoint, the most straightforward generalization consists of looking at the second, third neighbors, and so on. These are sometimes called the shells of a node. In this way, we may consider ApEn of the sequences generated by the number of (deterministic) walks of a given length. A variety of choices is then available: to look at the sequence of the number of walks of growing length, to count the number of walks starting from different nodes, etc. Remarkably, this allows us to associate various sequences with each node, which can be then compared, averaged, etc. The gain is the possibility of introducing network parameters for quantifying the disorder in the cycle structure of the graph. Graphs with a particularly disordered cycle structure, such as the controllable graphs introduced in [42], are expected to have higher ApEn of their walks.

If instead of combinatorial criteria, we aim at a more algebraic perspective, a first choice consists of taking the spectrum of a matrix that represents the network faithfully, like the adjacency matrix or a Laplacian. Indeed the spectrum of a network is a graph invariant and a naturally ordered sequence. A very superficial analysis based on Fig. 5 suggests that ApEn does not contain valuable information, or at least information that is not easy to interpret. Hence, it remains an open problem to determine what kind of network properties are identified by computing ApEn of spectra and whether this quantity grasps something about different matrix ensembles.

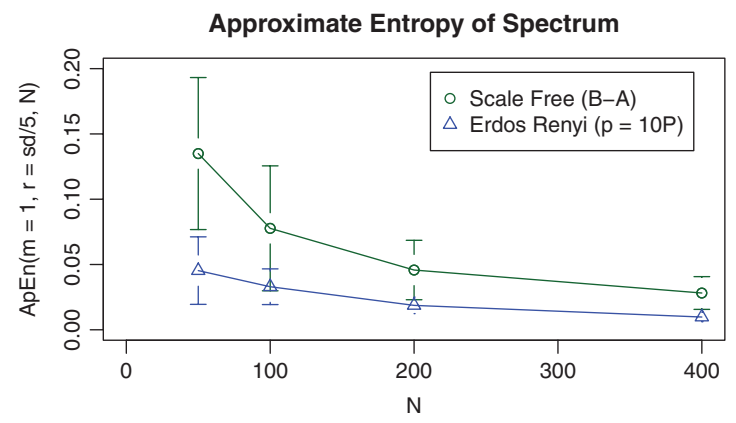

FIG. 5. (Color online) The approximate entropy of the adjacency spectrum. The figure illustrates ApEn for the Barabási-Albert scalefree networks $(\gamma=3)$ and for ordinary Erdös-Rényi graphs, where the probability of each edge is 10 times the critical probability for the graph to be (almost surely) connected. 


\section{ACKNOWLEDGMENTS}

The authors would like to thank the anonymous referees for their interesting comments. L.L. acknowledges hospitality from University of Oxford and University College London, where part of this research was conducted. J.W. is supported by EPSRC through a CoMPLEX Ph.D. studentship. L.L. acknowledges financial support from the MEC and Comunidad de Madrid (Spain) through Projects No. FIS2009-13690 and No. S2009ESP-1691. S.S. is supported by the Royal Society and A.E.T. by a Heller Research Fellowship.
[1] J.-P. Eckmann and D. Ruelle, Rev. Mod. Phys. 57, 617 (1985).

[2] M. E. J. Newman, SIAM Rev. 45, 167 (2003).

[3] M. E. J. Newman, Networks (Oxford University Press, 2010).

[4] J. Bascompte, P. Jordano, and J. M. Olesen, Science 312, 431 (2006).

[5] U. Bastolla, M. A. Fortuna, A. Pascual-García, A. Ferrera, B. Luque, and J. Bascompte, Nature (London) 458, 1018 (2009).

[6] S. Johnson, J. J. Torres, J. Marro, and M. A. Muñoz, Phys. Rev. Lett. 104, 108702 (2010).

[7] G. Bianconi, P. Pinb, and M. Marsili, Proc. Natl. Acad. Sci. USA 106, 28 (2009).

[8] M. Boguñá, R. Pastor-Satorras, and A. Vespignani, Phys. Rev. Lett. 90, 028701 (2003).

[9] R. Pastor-Satorras and A. Vespignani, Evolution and Structure of the Internet: A Statistical Physics Approach (Cambridge University Press, 2004).

[10] R. Pastor-Satorras and A. Vespignani, Phys. Rev. Lett. 86, 3200 (2001).

[11] G. Bianconi, Europhys. Lett. 81, 28005 (2008).

[12] G. Bianconi, Phys. Rev. E 79, 036114 (2009).

[13] S. L. Braustein, S. Gosh, and S. Severini, Ann. Comb. 10, 291 (2006).

[14] L. Donetti, P. I. Hurtado, and M. A. Muñoz, Phys. Rev. Lett. 95, 188701 (2005).

[15] F. Passerini and S. Severini, IJATS 1, 4 (2009).

[16] M. Dehmer and A. Mowshowitz, Inf. Sci. 181, 57 (2011).

[17] J. Gómez-Gardeñes and V. Latora, Phys. Rev. E 78, 065102(R) (2008).

[18] S. M. Pincus, Proc. Natl. Acad. Sci. USA 88, 2297 (1991).

[19] L. Lacasa, B. Luque, F. Ballesteros, J. Luque and J. C. Nuno, Proc. Natl. Acad. Sci. USA 105, 4973 (2008).

[20] B. Luque, L. Lacasa, F. Ballesteros, and J. Luque, Phys. Rev. E 80, 046103 (2009).

[21] S. M. Pincus, Methods Enzymol. 240, 68 (1994).

[22] S. M. Pincus, Chaos 5, 110 (1995).
[23] S. M. Pincus and B. H. Singer, Proc. Natl. Acad. Sci. USA 93, 2083 (1996).

[24] A. L. Rukhin, J. Appl. Probab. 37, 88 (2000).

[25] S. M. Pincus and R. E. Kalman, Proc. Natl. Acad. Sci. U.S.A. 101, 13709 (2004).

[26] J. Bhattacharya, Acta Neurobiol. Exp. 60, 4 (2000).

[27] J. W. Sleigh and J. Donovan, Br. J. Anaesth. 82(5), 666 (1999).

[28] A. Tripathi and S. Vijay, Discrete Math. 265, 417 (2003).

[29] A. Tripathi, S. Venugopalan, and D. B. West, Discrete Math. 310, 843 (2010).

[30] J. M. Burns, Ph.D. thesis, Massachusetts Institute of Technology, 2007.

[31] B. Pittel, J. Comb. Theory, Ser. A 88, 123 (1999).

[32] T. M. Rao, J. Comb. Theory, Ser. B 17, 19 (1974).

[33] A.-L. Barabási and R. Albert, Science 286, 509 (1999).

[34] B. Luque, L. Lacasa, F. J. Ballesteros, and A. Robledo, PLoS ONE 6(9), e22411 (2011).

[35] G. Ghoshal and A.-L. Barabási, Nature Commun. 2, 394 (2011).

[36] L. Lacasa, B. Luque, J. Luque, and J. C. Nuno, Europhys. Lett. 86, 30001 (2009).

[37] L. Lacasa and R. Toral, Phys. Rev. E 82, 036120 (2010).

[38] G. Gutin, T. Mansour, and S. Severini, Physica A 390, 12 (2011).

[39] S. F. Chin, A. E. Teschendorff, J. C. Marioni, Y. Wang, N. L. Barbosa-Morais, N. P. Thorne, J. L. Costa, S. E. Pinder, M. A. van de Wiel, A. R. Green, I. O. Ellis, P. L. Porter, S. Tavaré, J. D. Brenton, B. Ylstra, and C. Caldas, Genome Biol. 8, R215 (2007)

[40] A. E. Teschendorff and S. Severini, BMC Systems Biology 4, 104 (2010).

[41] H. G. Russnes, H. K. Vollan, O. C. Lingjaerde, A. Krasnitz, P. Lundin, B. Naume, T. Sørlie, E. Borgen, I. H. Rye, A. Langerød, S. F. Chin, A. E. Teschendorff, P. J. Stephens, S. Månér, E. Schlichting, L. O. Baumbusch, R. Kåresen, M. P. Stratton, M. Wigler, C. Caldas, A. Zetterberg, J. Hicks, and A. L. Børresen-Dale, Sci. Transl. Med. 2, 38ra47 (2010).

[42] C. Godsil and S. Severini, Phys. Rev. A 81, 052316 (2010). 